\title{
Solarium Use and Risk for Malignant Melanoma: Meta-analysis and Evidence-based Medicine Systematic Review
}

\author{
BARBARA BURGARD ${ }^{1,2^{*}}$, JAKOB SCHÖPE ${ }^{1,3^{*}}$, ISABEL HOLZSCHUH ${ }^{1,2}$, CLAUDIA SCHIEKOFER ${ }^{1,2}$, \\ SANDRA REICHRATH ${ }^{1,2}$, WAGENPFEIL STEFAN ${ }^{1,3}$, STEFAN PILZ ${ }^{4,5}$, JOSE ORDONEZ-MENA ${ }^{6,7,8}$, \\ WINFRIED MÄRZ ${ }^{9,10,11}$, THOMAS VOGT ${ }^{1,2}$ and JÖRG REICHRATH ${ }^{1,2}$ \\ ${ }^{1}$ Center for Clinical and Experimental Photodermatology, and ${ }^{3}$ Institute for Medical Biometry, \\ Epidemiology and Medical Informatics, Saarland University, Campus Homburg, Homburg, Germany; \\ ${ }^{2}$ Department of Dermatology, The Saarland University Hospital, Homburg, Germany; \\ ${ }^{4}$ Department of Epidemiology and Biostatistics, EMGO Institute for Health and Care Research, \\ VU University Medical Center, Amsterdam, the Netherlands; \\ ${ }^{5}$ Department of Internal Medicine, Division of Endocrinology and Metabolism, and \\ ${ }^{10}$ Clinical Institute of Medical and Chemical Laboratory Diagnostics, Medical University of Graz, Graz, Austria; \\ ${ }^{6}$ Division of Clinical Epidemiology and Aging Research, \\ German Cancer Research Center (DKFZ), Heidelberg, Germany; \\ ${ }^{7}$ Network Aging Research (NAR), Heidelberg University, Heidelberg, Germany; \\ ${ }^{8}$ Nuffield Department of Primary Care Health Sciences, University of Oxford, Oxford, U.K.; \\ ${ }^{9}$ Medical Clinic V (Nephrology, Hypertensiology, Rheumatology, Endocrinology, Diabetology), \\ Medical Faculty Mannheim, Ruperto-Carola University of Heidelberg, Heidelberg, Germany; \\ ${ }^{11}$ Synlab Medical Center of Human Genetics Mannheim, Mannheim, Germany
}

\begin{abstract}
Background: There is an ongoing debate whether solarium use (indoor tanning/artificial UV) may increase the risk for primary cutaneous malignant melanoma. Aim: A systematic literature search was conducted using MEDLINE and ISI Web of Science. Included studies were critically assessed regarding their risk of bias, and methodological shortcomings. Levels of evidence and grades of recommendation were determined according to guidelines of the Oxford Centre for Evidence-Based Medicine. Summary risk estimates and 95\% confidence intervals for four different outcomes (ever exposure, exposure at younger age, high/low exposure vs. non-exposure) were derived from random-effects meta-analyses to account for possible heterogeneity across studies. Results: Two cohort and twenty-nine case-control
\end{abstract}

*These Authors contributed equally to this study.

Correspondence to: Prof. Dr. med. J. Reichrath, Department of Dermatology, The Saarland University Hospital, Kirrbergerstr. 100 66421 Homburg, Germany, Tel: +49 068411623802, e-mail: Joerg.reichrath@uks.eu

Key Words: Malignant melanoma, solarium, UV, review. studies were eligible. Overall, quality of included studies was poor as a result of severe limitations, including possible recall and selection bias, and due to lack of interventional trials. Summary risk estimates suggested a weak association (odds ratio $(O R)=1.19,95 \%$ confidence interval $(C I)=1.04$ 1.35, $p=0.009$ ) for ever-exposure to $U V$ radiation from a solarium with melanoma risk. However, sensitivity analyses did not show an association for studies from Europe $(O R=1.10 ; 95 \% C I=0.95-1.27, p=0.218)$, studies with low risk of bias $(O R=1.15 ; 95 \% C I=0.94-1.41, p=0.179)$, and studies conducted after 1990 (OR 1.09; 95\%CI=0.93-1.29, $p=0.295)$. Moreover, moderate associations were found for first exposure to $U V$ radiation from a solarium at younger age ( $<25$ years) and high exposure ( $>10$ sessions in lifetime) with melanoma risk. However, for all outcomes analyzed, overall study quality and resulting levels of evidence (3a-) and grades of recommendation $(D)$ were low due to lack of interventional studies and severe limitations including unobserved or unrecorded confounding. Conclusion: Current scientific knowledge is mainly based on observational studies with poor quality data, which report associations but do not prove causality. At present, there is no convincing evidence that moderate/responsible solarium use increases melanoma risk. 
Sunlight represents an important pre-requisite for the development of life on earth and for human evolution $(1,2)$. Of particular importance is the ultraviolet (UV) range (UVC: 200-280 nm; UV-B: 280-315 nm; UV-A: 315-400 nm) of solar radiation, because exposure to solar or artificial UV exerts both positive and negative effects on human health (1$5)$. While some of the beneficial UV effects are due to the UV-B-mediated cutaneous synthesis of vitamin D (1-5), hazardous effects include the initiation and promotion of skin photocarcinogenesis (6). The relevance of the UV-B spectrum in promoting non-melanoma skin cancer (risk factor: high cumulative exposure, via e.g. induction of DNA mutations) and melanoma (risk factor: high intermittent exposure, e.g. sunburn, most importantly in childhood) is generally accepted. Laboratory and animal studies have more recently suggested a possible additional contribution of the UV-A spectrum to skin photocarcinogenesis $(5,6)$. Malignant melanoma represents the most aggressive form of skin cancer. While melanoma death rates had more than doubled in light-skinned populations between 1955 and 1985, decreases in melanoma mortality rates were observed from 1985-1990 in Australia, the United States and in many European countries (7).

In this context, the beneficial and hazardous effects of solarium use, in particular the relevance of solarium use as a potential risk factor for malignant melanoma, are still a matter of debate. Since the 1980s, solarium use has been widely practised in Western and Northern Europe, Canada and the United States, and since 2000 it has become common even in sun-rich countries such as Australia (8). Modern solarium devices mainly emit UV-A radiation, only a small fraction $(<5 \%)$ of radiation is emitted in the UV-B range (8). Many studies have investigated the impact of indoor tanning on melanoma risk (8-59), however, most of them have been criticized for limitations, unbalanced view, errors or incorrectnesses $(11,17)$. While some reports suggest that solarium use may increase melanoma risk (e.g. 19, 29, 32), other investigations found no or even a protective effect $(e . g$. 20-22, 24, 35). In 2009, the International Agency for Research on Cancer (IARC) classified the complete ultraviolet spectrum (including UV-A) and solarium devices as Group One carcinogens to humans (9). To improve our present understanding about the relevance of solarium use as a potential risk factor for malignant melanoma, we performed a meta-analysis and evidencebased systematic review of the literature.

\section{Materials and Methods}

This meta-analysis was performed in accordance with the Preferred Reporting Items for Systematic Reviews and Meta-analyses (PRISMA) (60), and the Meta-analysis of Observational studies in Epidemiology (MOOSE) guidelines (61).
Search strategy and inclusion criteria. The following terms and their variations were considered to be synonymous with solarium: indoor tanning, sunbed, sunlamp, artificial UV exposure, and non-solar UVexposure. Relevant publications were searched independently by four Authors (BB, IH, SR, JR) in MEDLINE (from 1946) and ISI Web of Science (from 1945) using combinations of the following keywords: "sunbed", "sunlamp", "solarium", "solaria", "artificial UV", "artificial light", "UV”, "indoor tanning", "tanning bed", "tanning parlour", "tanning salon", "tanning booth", "skin cancer", and "melanoma". Identified articles including reviews were cross-referenced for articles missed by database search. Inclusion criteria were study type/content (interventional, cohort, case-control studies published up until January 15, 2016, which reported an association between exposure to UV radiation from a solarium and cutaneous malignant melanoma), types of outcome measures (for retrospective and prospective studies: development of melanoma) and no language restrictions. In case of duplicate samples, the most recent study was included. Exclusion criteria were defined accordingly.

Outcome measures. Main outcome measures were defined as "everexposure to UV radiation from a solarium (yes)", "high/low exposure to UV radiation from a solarium" and "first exposure to UV radiation from a solarium at younger age". All exposures were compared against non-exposure. Data were independently extracted by three Authors (BB, JR and JS), and transferred to a data collection sheet that considered relevant parts of the Cochrane Consumers and Communication Review Group's data extraction template (62).

Assessment of risk of bias. Risk of bias was assessed independently by two authors (JR, JS) using a modification of the NewcastleOttawa Quality Assessment Scale (MNOS;) (63). Moreover, potential biases derived from methodological shortcomings were evaluated by SW and JS. Consensus regarding the grading was sought and disagreements were discussed with a third Author (JR). Finally, the risk of bias was indicated as low risk of bias (MNOS $>4$ ) and high risk of bias (MNOS $\leq 4)$.

Level of evidence and grade of recommendation. For main outcomes, level of evidence and grade of recommendation were determined according to the Oxford Centre for Evidence-based Medicine (64).

Statistical analysis. Reported risk estimates [odds ratio (OR) or hazard ratio (HR)] across included studies were not consistently adjusted for confounders causing methodological heterogeneity. Accordingly, crude risk estimates (only OR) and their 95\% confidence intervals (CI) were calculated from studies' contingency tables, or were taken from the original articles. Additionally, adjusted risk estimates were obtained from original articles, and were used to compare crude summary risk estimates with adjusted summary risk estimates. After log-transforming all estimates, standard errors (SE) were determined by subtracting the lower logtransformed CI boundary from the upper log-transformed CI boundary, and dividing its sum by 3.92 . Heterogeneity ( $Q$ and $\mathrm{I}^{2}$ statistics) was taken into account by performing a random-effects meta-analysis. Summary risk estimates of random-effects models from maximum likelihood estimations are shown as forest plots for studies with a consistent risk estimate (OR). Potential publication bias was assessed using funnel plot and Egger's test. All analyses were conducted using the metafor-package in R 3.2.1. 
Sensitivity analysis. Sensitivity analysis was conducted to verify the robustness of pooled results, and to explore possible causes of heterogeneity. Accordingly, subgroup analyses were performed for study design (case-control study, cohort study), geographic regions (United States of America and Canada, Australia, Europe), trends over time (year of recruitment $<1990,1991-1999, \geq 2000$ ), and risk of bias (high risk, low risk).

\section{Results}

Literature search. The literature search process (described in Figure 1) identified 41 studies that investigated an association between solarium use and melanoma risk. Six studies were excluded because they did not report any risk estimates for this association (50-55). Duplicate samples were found in four studies, and were therefore redundant (56-59). Finally, our literature search identified two cohort and 29 case-control studies which were eligible for metaanalysis (19-49).

Study characteristics. Characteristics of the included studies are shown in Table I. Most studies were conducted in Europe (64.5\%), followed by North America (29.0\%), and Australia $(6.5 \%)$. Samples were mainly recruited before $2000(80.0 \%)$, and differed in age and gender distributions. Overall, included studies comprised 11,706 malignant melanoma cases and 93,236 controls regarding the association between ever exposure to UV radiation from a solarium and melanoma risk (see Table II).

Assessment of study quality, level of evidence and grade of recommendation. The overall quality of studies and the resulting evidence levels were low due to the lack of interventional trials and severe limitations (including unobserved or unrecorded confounding) of many of the observational studies, which might cause a high risk of bias (Tables II and III). It should be emphasized that the results of these cohort and case-control studies represent associations and do not prove causality. Remarkably, in all studies most likely risk of bias resulted in an overestimation of melanoma risk (supplemental file available from the authors upon request). Scores on the modified Newcastle-Ottawa Quality Assessment Scale were on average low, as $67.7 \%$ of the 31 included cohort and case-control studies scored less than four stars (supplemental file available from the authors upon request). Assessing all individual studies according to recommendations of the Oxford Centre for Evidence-based Medicine, we defined the association of ever-exposure, first exposure at younger age and high/low exposure to UV radiation from a solarium with melanoma risk as level four of evidence (poor quality cohort and case-control studies) and grade D of recommendation (supplemental file available from the authors upon request). Only a minority of studies reported ORs that were adjusted for the same confounding factors. As

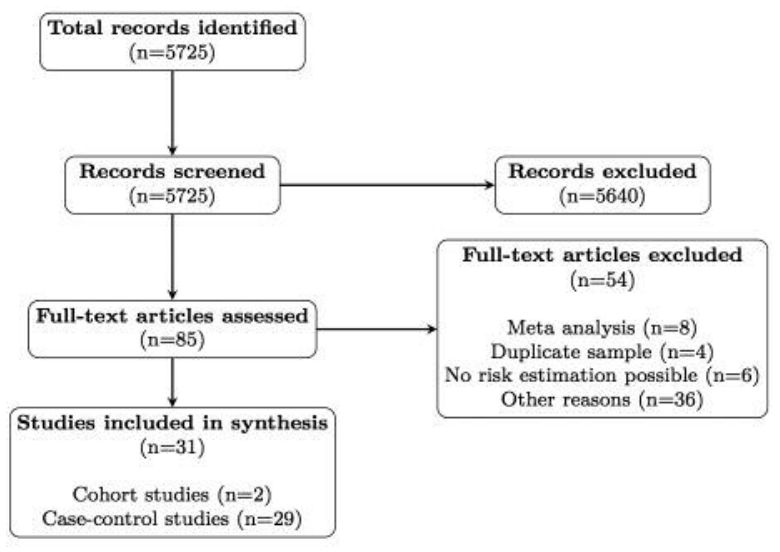

Figure 1. Flowchart of the literature search process.

many as $35.5 \%(n=11)$ of all the included studies did not account for a single confounder. The remaining studies $(n=20)$ adjusted mainly for age $(n=15)$, sex $(n=11)$, and skin color $(n=11)$. Fewer studies adjusted for hair colour $(n=10)$, sun exposure $(n=8)$, sunburns $(n=8)$, family history of melanoma $(n=7)$, naevi $(n=7)$, freckles $(n=5)$ and education $(n=5)$. Moreover, individual confounders were assessed across the included studies differently, and were only partly comparable. Overall, we observed a relatively high heterogeneity across included studies (e.g. ever-exposure: $\mathrm{I}^{2}=75.98 \%$ ), and thus performed a random-effects meta-analysis.

Association of ever-exposure to UV radiation from a solarium with melanoma risk. The summary risk estimate of the randomeffects meta-analysis for all studies (cohort and case-control studies combined, as seen in Figures 2-4) showed a statistically significant weak association for ever-exposure to UV radiation from a solarium with melanoma risk compared with nonexposure (as seen in Figure 2, overall relative risk $=1.19 ; 95 \%$ $\left.\mathrm{CI}=1.05-1.34 ; \mathrm{Q}(30)=114.33 ; p<0.001 ; \mathrm{I}^{2}=74.55 \%\right)$. Exclusion of the study by Nielsen et al. (40), which reported a HR instead of an OR, altered results only slightly (Table III; OR $=1.19$; 95\% CI=1.04-1.35; Q(29)=114.33; $\left.p<0.001 ; \mathrm{I}^{2}=75.98 \%\right)$. The funnel plot did not show evidence of publication bias (Figure 5, Egger's test; $p=0.169$ ).

Sensitivity analyses yielded results inconsistent with our main finding. Subgroup analyses did not show statistically significant associations when separating for geographic region (studies performed in Europe, Figure 2 and Table III; $\mathrm{OR}=1.10 ; 95 \%$ CI $0.95-1.27 ; \mathrm{Q}(18)=49.39 ; p<0.001$; $\mathrm{I}^{2}=60.15 \%$ ), risk of bias (studies with low risk of bias, Figure 3 and Table III; $\mathrm{OR}=1.15 ; 95 \% \quad \mathrm{CI}=0.94-1.41$; $\mathrm{Q}(10)=29.63 ; p=0.001 ; \mathrm{I}^{2}=66.30 \%$ ), and trends over time (studies conducted after 1990, Figure 4 and Table III; $\mathrm{OR}=1.09 ; \quad 95 \% \quad \mathrm{CI}=0.93-1.29 ; \quad \mathrm{Q}(15)=72.97 ; \quad p<0.001$; 
Table I. Characteristics of included studies $(n=31)$.

\begin{tabular}{|c|c|c|c|c|c|c|c|}
\hline Study (Reference number) & Design & $\begin{array}{l}\text { Recruitment } \\
\text { period }\end{array}$ & Matching & $\begin{array}{l}\text { Gender } \\
(\mathrm{f} / \mathrm{m} \%)\end{array}$ & $\begin{array}{c}\text { Age } \\
\text { (range in years) }\end{array}$ & Ethnicity & $\begin{array}{l}\text { Place of } \\
\text { recruitment }\end{array}$ \\
\hline Adam et al. (19) & $\mathrm{CC}$ & 1971-1976 & FM & $100 \% / 0 \%$ & $15-49$ & Caucasian & GBR \\
\hline Autier et al. (20) & $\mathrm{CC}$ & $1991-n / s$ & FM & $\mathrm{n} / \mathrm{s}$ & $20-n / s$ & Caucasian & GER, FRA, BEL \\
\hline Bataille et al. (20) & $\mathrm{CC}$ & 1989-1993 & FM & $60.3 \% / 39.7 \%$ & $16-75$ & $\mathrm{n} / \mathrm{s}$ & GBR \\
\hline Bataille et al. (21) & $\mathrm{CC}$ & 1998-2001 & FM & $64.5 \% / 35.5 \%$ & $18-49$ & Caucasian & BEL, NLD, FRA, SWE, GBR \\
\hline Chen et al. (23) & $\mathrm{CC}$ & 1987-1989 & FM & $\mathrm{n} / \mathrm{s}$ & $\mathrm{n} / \mathrm{s}$ & Caucasian & USA \\
\hline Clough-Gorr et al. (24) & $\mathrm{CC}$ & 1995-1998 & FM & $48.1 \% / 51.9 \%$ & $20-69$ & $\mathrm{n} / \mathrm{s}$ & USA \\
\hline Cust et al. (25) & $\mathrm{CC}$ & 2000-2002 & FM & $60.1 \% / 39.9 \%$ & $18-39$ & Caucasian & AUS \\
\hline Dunn-Lane et al. (26) & $\mathrm{CC}$ & 1985-1986 & FM & $71.0 \% / 29.0 \%$ & $15-82$ & $\mathrm{n} / \mathrm{s}$ & IRL \\
\hline Elliott et al. (27) & $\mathrm{CC}$ & 2000-2005 & FM & $59.6 \% / 40.4 \%$ & $17-76$ & $\mathrm{n} / \mathrm{s}$ & GBR \\
\hline Elwood et al. (28) & $\mathrm{CC}$ & 1981-1984 & IM & $70.0 \% / 30.0 \%$ & $18-82$ & $\mathrm{n} / \mathrm{s}$ & GBR \\
\hline Farley et al. (29) & $\mathrm{CC}$ & 2001-2013 & NM & $56.5 \% / 43.5 \%$ & $18-50$ & $\mathrm{n} / \mathrm{s}$ & USA \\
\hline Fears et al. (30) & $\mathrm{CC}$ & 1991-1992 & FM & $\mathrm{n} / \mathrm{s}$ & $20-79$ & Caucasian & USA \\
\hline Garbe et al. (31) & $\mathrm{CC}$ & $1983-1990$ & NM & $\mathrm{n} / \mathrm{s}$ & $\mathrm{n} / \mathrm{s}$ & $\mathrm{n} / \mathrm{s}$ & AUT, GER, CHE \\
\hline Han et al. (32) & $\mathrm{NCC}$ & $1989-2000$ & IM & $100 \% / 0 \%$ & $43-68$ & Caucasian & USA \\
\hline Holly et al. (33) & $\mathrm{CC}$ & 1981-1986 & FM & $100 \% / 0 \%$ & $25-59$ & Caucasian & USA \\
\hline Holman et al. (34) & $\mathrm{CC}$ & 1980-1981 & FM & $\mathrm{n} / \mathrm{s}$ & $\mathrm{n} / \mathrm{s}$ & $\mathrm{n} / \mathrm{s}$ & AUS \\
\hline Kaskel et al. (35) & $\mathrm{CC}$ & 1997-1999 & NM & $50.5 \% / 49.5 \%$ & $19-90$ & Caucasian & GER \\
\hline Landi et al. (36) & $\mathrm{CC}$ & 1994-1999 & FM & $51.4 \% / 48.6 \%$ & $17-77$ & Caucasian & ITA \\
\hline Lazovich et al. (37) & $\mathrm{CC}$ & 2004-2007 & FM & $59.7 \% / 40.3 \%$ & $25-59$ & Caucasian & USA \\
\hline MacKie et al. (38) & $\mathrm{CC}$ & 1987 & IM & $64.6 \% / 35.4 \%$ & $11-n / s$ & $\mathrm{n} / \mathrm{s}$ & GBR \\
\hline Naldi et al. (39) & $\mathrm{CC}$ & 1992-1995 & NM & $\mathrm{n} / \mathrm{s}$ & $\mathrm{n} / \mathrm{s}$ & $\mathrm{n} / \mathrm{s}$ & ITA \\
\hline Nielsen et al. (40) & $\mathrm{CO}$ & 1990-1992 & $\mathrm{n} / \mathrm{a}$ & $100 \% / 0 \%$ & $25-64$ & $\mathrm{n} / \mathrm{s}$ & SWE \\
\hline Østerlind et al. (41) & $\mathrm{CC}$ & $1982-1985$ & FM & $\mathrm{n} / \mathrm{s}$ & $20-79$ & $\mathrm{n} / \mathrm{s}$ & DNK \\
\hline Swerdlow et al. (42) & $\mathrm{CC}$ & 1979-1984 & FM & $\mathrm{n} / \mathrm{s}$ & $15-84$ & $\mathrm{n} / \mathrm{s}$ & GBR \\
\hline Ting et al. (43) & $\mathrm{CC}$ & $\mathrm{n} / \mathrm{s}$ & $\mathrm{n} / \mathrm{s}$ & $61.2 \% / 38.8 \%$ & $\mathrm{n} / \mathrm{s}$ & Caucasian & USA \\
\hline Veierød et al. (44) & $\mathrm{CO}$ & 1991-1992 & $\mathrm{n} / \mathrm{a}$ & $100 \% / 0 \%$ & $30-50$ & $\mathrm{n} / \mathrm{s}$ & NOR, SWE \\
\hline Walter et al. (45) & $\mathrm{CC}$ & 1984-1986 & FM & $53.0 \% / 47.0 \%$ & $20-69$ & $\mathrm{n} / \mathrm{s}$ & CAN \\
\hline Westerdahl et al. (46) & $\mathrm{CC}$ & $1988-1990$ & IM & $51.4 \% / 48.6 \%$ & $15-75$ & $\mathrm{n} / \mathrm{s}$ & SWE \\
\hline Westerdahl et al. (47) & $\mathrm{CC}$ & 1995-1997 & IM & $\mathrm{n} / \mathrm{s}$ & $16-80$ & $\mathrm{n} / \mathrm{s}$ & SWE \\
\hline Wolf et al. (48) & $\mathrm{CC}$ & 1993-1994 & NM & $57.6 \% / 42.4 \%$ & $15-83$ & $\mathrm{n} / \mathrm{s}$ & AUT \\
\hline Zanetti et al. (49) & $\mathrm{CC}$ & 1984-1986 & NM & $54.5 \% / 45.5 \%$ & $17-92$ & $\mathrm{n} / \mathrm{s}$ & ITA \\
\hline
\end{tabular}

AUS: Australia, AUT: Austria, BEL: Belgium, CAN: Canada, CC: case-control study, CHE: Switzerland, CO: cohort study, DNK: Denmark, f: female, FM: frequency matching, FRA: France, GER: Germany, GBR: United Kingdom of Great Britain and Northern Ireland, HRV: Croatia, IM: individual matching, IRL: Ireland, ITA: Italy, m: male, n/a: not applicable, NCC: nested case-control study, NLD: The Netherlands, NM: no matching, NOR: Norway, n/s: not stated, SWE: Sweden, USA: United States of America. Rounding errors may occur in data table. Gender proportions are approximated for total sample sizes, and may differ from original data.

$\mathrm{I}^{2}=79.51 \%$ ). According to the Oxford Centre for Evidencebased Medicine, for the outcome ever-exposure to UV radiation from a solarium, we determined an evidence level of $3 a-$ - (systematic review of poor quality cohort and casecontrol studies) and a grade D of recommendation.

Association of first exposure to UV radiation from a solarium at young age with melanoma risk. Thirteen included studies investigated a possible association between age at first use of a solarium and melanoma risk. However, only four studies reported a risk estimate for the same age threshold $(<25$ years). For consistency, a meta-analysis was solely performed with these four studies. The summary risk estimate indicated a statistically significant moderate association between first exposure to UV radiation from a solarium before age 25 years and melanoma risk (Table III; OR $=1.59$; 95\% $\mathrm{CI}=1.38-1.83$; $\left.\mathrm{Q}(3)=1.06 ; p=0.787 ; \mathrm{I}^{2}=0.00 \%\right)$. According to the Oxford Centre for Evidence-based Medicine, for the outcome first exposure to UV radiation from a solarium at young age" we determined an evidence level of 3a- (systematic review of poor quality cohort and case-control studies) and a grade D of recommendation.

Association of high/low exposure to UV radiation from a solarium with melanoma risk. Several included studies $(n=15)$ determined possible dose-response relationships between exposure to UV radiation from a solarium and melanoma risk. Seven out of these studies used a consistent 
Table II. Risk estimates for included case-control and cohort studies ( $n=31)$.

\begin{tabular}{|c|c|c|c|c|c|c|c|}
\hline \multirow[b]{2}{*}{ Study } & \multicolumn{2}{|c|}{ Sample size (n) } & \multirow[b]{2}{*}{ Cases } & \multirow[b]{2}{*}{ Controls } & \multicolumn{2}{|c|}{ Ever exposure $v s$. non-exposure } & \multirow[b]{2}{*}{ Adjustment } \\
\hline & Cases & Controls & & & Crude OR $(95 \% \mathrm{CI})$ & Adjusted OR/HR (95\% CI) & \\
\hline Adam et al. (19) & 111 & 342 & $9 / 102$ & $11 / 331$ & $2.66(1.07-6.59)^{1}$ & $\mathrm{n} / \mathrm{s}$ & $\mathrm{n} / \mathrm{a}$ \\
\hline Autier et al. (20) & 420 & 447 & $110 / 310$ & $120 / 327$ & $0.97(0.72-1.31)^{2}$ & $\mathrm{n} / \mathrm{s}$ & $\mathrm{n} / \mathrm{a}$ \\
\hline Bataille et al. (21) & 413 & 416 & $95 / 314$ & $106 / 306$ & $0.87(0.64-1.20)^{1}$ & $1.19(0.84-1.68)$ & $\mathrm{a}, \mathrm{p}$ \\
\hline Bataille et al. (22) & 597 & 622 & $315 / 282$ & $354 / 268$ & $0.85(0.67-1.06)^{2}$ & $0.90(0.71-1.14)$ & $\mathrm{a}, \mathrm{p}, \mathrm{q}$ \\
\hline Chen et al. (23) & 624 & 512 & $141 / 483$ & $95 / 417$ & $1.28(0.96-1.71)^{1}$ & $1.13(0.82-1.54)$ & $\mathrm{a}, \mathrm{p}, \mathrm{q}, \mathrm{s}$ \\
\hline Clough-Gorr et al. (24) & 423 & 678 & $267 / 156$ & $460 / 218$ & $0.81(0.63-1.05)^{1}$ & $1.22(0.83-1.80)$ & $\mathrm{a}, \mathrm{e}, \mathrm{f}, \mathrm{g}, \mathrm{p}, \mathrm{s}, \mathrm{t}$ \\
\hline Cust et al. (25) & 604 & 479 & $137 / 467$ & $84 / 395$ & $1.38(1.02-1.87)^{1}$ & $1.41(1.01-1.96)$ & $\mathrm{a}, \mathrm{c}, \mathrm{e}, \mathrm{l}, \mathrm{p}, \mathrm{q}, \mathrm{s}, \mathrm{t}$ \\
\hline Dunn-Lane et al. (26) & 100 & 100 & $17 / 83$ & $15 / 85$ & $1.16(0.54-2.48)^{1}$ & $\mathrm{n} / \mathrm{s}$ & n/a \\
\hline Elliott et al. (27) & 959 & 513 & $441 / 414$ & $225 / 258$ & $1.22(0.98-1.53)^{1}$ & $1.06(0.83-1.36)$ & $\mathrm{a}, \mathrm{c}, \mathrm{e}, \mathrm{p}, \mathrm{s}, \mathrm{t}$ \\
\hline Elwood et al. (28) & 83 & 83 & $15 / 68$ & $12 / 71$ & $1.31(0.57-2.99)^{1}$ & $\mathrm{n} / \mathrm{s}$ & $\mathrm{n} / \mathrm{a}$ \\
\hline Farley et al. (29) & 265 & 195 & $140 / 125$ & $70 / 125$ & $2.00(1.37-2.92)^{1}$ & $\mathrm{n} / \mathrm{s}$ & $\mathrm{n} / \mathrm{a}$ \\
\hline Fears et al. (30) & 718 & 945 & $188 / 530$ & $282 / 662$ & $0.83(0.67-1.03)^{1}$ & $\mathrm{n} / \mathrm{s}$ & $\mathrm{n} / \mathrm{a}$ \\
\hline Garbe et al. (31) & 856 & 705 & $66 / 790$ & $50 / 655$ & $1.09(0.75-1.60)^{1}$ & $1.5(0.9-2.4)$ & $\mathrm{a}, \mathrm{g}, \mathrm{k}, \mathrm{l}, \mathrm{q}$ \\
\hline Han et al. (32) & 200 & 804 & $42 / 140$ & $87 / 625$ & $2.16(1.43-3.25)^{1}$ & $2.06(1.30-3.26)$ & $\mathrm{a}, \mathrm{e}, \mathrm{o}, \mathrm{s}, \mathrm{r}, \mathrm{v}$ \\
\hline Holly et al. (33) & 452 & 930 & $\mathrm{n} / \mathrm{s}$ & $\mathrm{n} / \mathrm{s}$ & $0.94(0.74-1.20)^{2}$ & $\mathrm{n} / \mathrm{s}$ & $\mathrm{n} / \mathrm{a}$ \\
\hline Holman et al. (34) & 511 & 511 & $\mathrm{n} / \mathrm{s}$ & $\mathrm{n} / \mathrm{s}$ & $1.1(0.6-1.8)^{2}$ & $\mathrm{n} / \mathrm{s}$ & $\mathrm{n} / \mathrm{a}$ \\
\hline Kaskel et al. (35) & 291 & 329 & $6 / 285$ & $21 / 308$ & $0.31(0.12-0.78)^{1}$ & $\mathrm{n} / \mathrm{s}$ & $\mathrm{n} / \mathrm{a}$ \\
\hline Landi et al. (36) & 183 & 179 & $32 / 150$ & $38 / 141$ & $0.79(0.47-1.34)^{1}$ & $1.3(0.7-2.4)$ & $\mathrm{a}, \mathrm{d}, \mathrm{m}, \mathrm{n}, \mathrm{p}, \mathrm{q}$ \\
\hline Lazovich et al. (37) & 1167 & 1101 & $734 / 433$ & $563 / 538$ & $1.62(1.37-1.92)^{1}$ & $1.74(1.42-2.14)$ & $\mathrm{a}, \mathrm{c}, \mathrm{d}, \mathrm{e}, \mathrm{f}, \mathrm{g}, \mathrm{h}, \mathrm{j}, \mathrm{p}, \mathrm{q}, \mathrm{r}, \mathrm{s}, \mathrm{u}$ \\
\hline MacKie et al. (38) & 280 & 280 & $33 / 247$ & $8 / 272$ & $4.54(2.06-10.02)^{1}$ & $1.22(0.54-2.73)$ & $\mathrm{f}, \mathrm{k}, \mathrm{o}, \mathrm{q}, \mathrm{r}$ \\
\hline Naldi et al. (39) & 542 & 538 & $30 / 512$ & $36 / 502$ & $0.82(0.50-1.35)^{1}$ & $0.78(0.45-1.37)$ & $\mathrm{a}, \mathrm{c}, \mathrm{d}, \mathrm{f}, \mathrm{g}, \mathrm{i}, \mathrm{k}, \mathrm{p}, \mathrm{q}, \mathrm{r}, \mathrm{x}$ \\
\hline Nielsen et al. (40) & 206 & 29,314 & $\mathrm{n} / \mathrm{s}$ & $\mathrm{n} / \mathrm{s}$ & $\mathrm{n} / \mathrm{s}$ & $1.17(0.79-1.72)$ & $\mathrm{b}, \mathrm{e}, \mathrm{f}, \mathrm{g}, \mathrm{k}, \mathrm{r}, \mathrm{u}, \mathrm{w}, \mathrm{x}$ \\
\hline Østerlind et al. (41) & 474 & 926 & $66 / 408$ & $167 / 759$ & $0.74(0.54-1.00)^{1}$ & $\mathrm{n} / \mathrm{s}$ & $\mathrm{n} / \mathrm{a}$ \\
\hline Swerdlow et al. (42) & 180 & 197 & $38 / 142$ & $10 / 110$ & $2.94(1.40-6.17)^{1}$ & $2.94(1.4-6.4)$ & $\mathrm{d}, \mathrm{g}, \mathrm{k}, \mathrm{q}, \mathrm{s}$ \\
\hline Ting et al. (43) & 79 & 1439 & $34 / 45$ & $453 / 986$ & $1.64(1.04-2.60)^{1}$ & $\mathrm{n} / \mathrm{s}$ & $\mathrm{n} / \mathrm{a}$ \\
\hline Veierød et al. (44) & 412 & 105,954 & $178 / 137$ & $40873 / 37854$ & $1.20(0.96-1.50)^{1}$ & $1.31(1.03-1.66)$ & $\mathrm{a}, \mathrm{g}, \mathrm{o}, \mathrm{q}, \mathrm{s}$ \\
\hline Walter et al. (45) & 583 & 608 & $152 / 431$ & $109 / 498$ & $1.61(1.22-2.13)^{1}$ & $1.54(1.16-2.05)$ & $\mathrm{a}, \mathrm{p}, \mathrm{t}$ \\
\hline Westerdahl et al. (46) & 400 & 640 & $115 / 282$ & $155 / 479$ & $1.26(0.95-1.67)^{1}$ & $1.3(0.9-1.8)$ & $\mathrm{e}, \mathrm{g}, \mathrm{k}, \mathrm{r}, \mathrm{x}$ \\
\hline Westerdahl et al. (47) & 571 & 913 & $250 / 319$ & $372 / 538$ & $1.13(0.92-1.40)^{1}$ & $1.2(0.9-1.6)$ & $\mathrm{g}, \mathrm{k}, \mathrm{q}, \mathrm{r}$ \\
\hline Wolf et al. (48) & 193 & 319 & $11 / 181$ & $16 / 300$ & $1.14(0.52-2.51)^{1}$ & $1.34(0.58-3.07)$ & $\mathrm{a}, \mathrm{p}$ \\
\hline Zanetti et al. (49) & 208 & 416 & $15 / 193$ & $21 / 395$ & $1.46(0.74-2.90)^{1}$ & $0.9(0.4-2.0)$ & $\mathrm{a}, \mathrm{c}, \mathrm{g}, \mathrm{r}, \mathrm{t}$ \\
\hline
\end{tabular}

n/a: Not applicable, n/s: not stated, a: age, b: blisters, c: education, d: eye color, e: family history of melanoma, f: freckles, g: hair color, h: income, i: marital status, j: moles, k: naevi, l: place of recruitment, m: presence of DN, n: propensity to tan, o: region of residence, p: sex, q: skin colour, r: sunburns, s: sun exposure, t: sun sensitivity, u: sunscreen use, v: susceptibility, w: ulcers, x: vacations. ${ }^{1}$ Calculated from contingency table, ${ }^{2}$ obtained from publication. Rounding error may occur in data table. Number of cases and controls from risk estimations may differ from total sample sizes due to missing data. Adjusted risk estimates (with max. number of confounders) were obtained from original articles. Nielsen et al. (40) reported an HR, others reported OR.

definition ( $>10$ sessions in lifetime) and were thus appropriate for meta-analysis. The pooled result of this analysis indicated a statistically significant moderate association for high exposure to UV radiation from a solarium with melanoma risk (Table III; OR $=1.43$; $95 \%$ $\left.\mathrm{CI}=1.17-1.74 ; \mathrm{Q}(6)=19.32 ; p=0.004 ; \mathrm{I}^{2}=60.87 \%\right)$. However, most of the pooled studies $(85.7 \%)$ had a high risk of bias. A meta-analysis with the same seven studies was performed for low exposure to UV radiation from a solarium (defined as $\leq 10$ sessions in lifetime) and did not show a statistically significant association (Table III; OR $=1.13$; 95\% CI=0.931.38; $\left.\mathrm{Q}(6)=17.06 ; p=0.009 ; \mathrm{I}^{2}=58.51 \%\right)$. According to the Oxford Centre for Evidence-based Medicine, for the outcome "high and low exposure to UV radiation from a solarium and melanoma risk" we found evidence level of $3 \mathrm{a}-$ (systematic review of poor quality cohort and casecontrol studies) and a grade $\mathrm{D}$ of recommendation.

\section{Discussion}

Several meta-analyses and reviews have already investigated the relevance of solarium use as a potential melanoma risk factor in recent years. However, most of them have been criticized for limitations, unbalanced view, errors, or incorrectnesses $(11,17)$. As an example, incorrectnesses in one of the main findings of the study of Boniol et al. (8) 


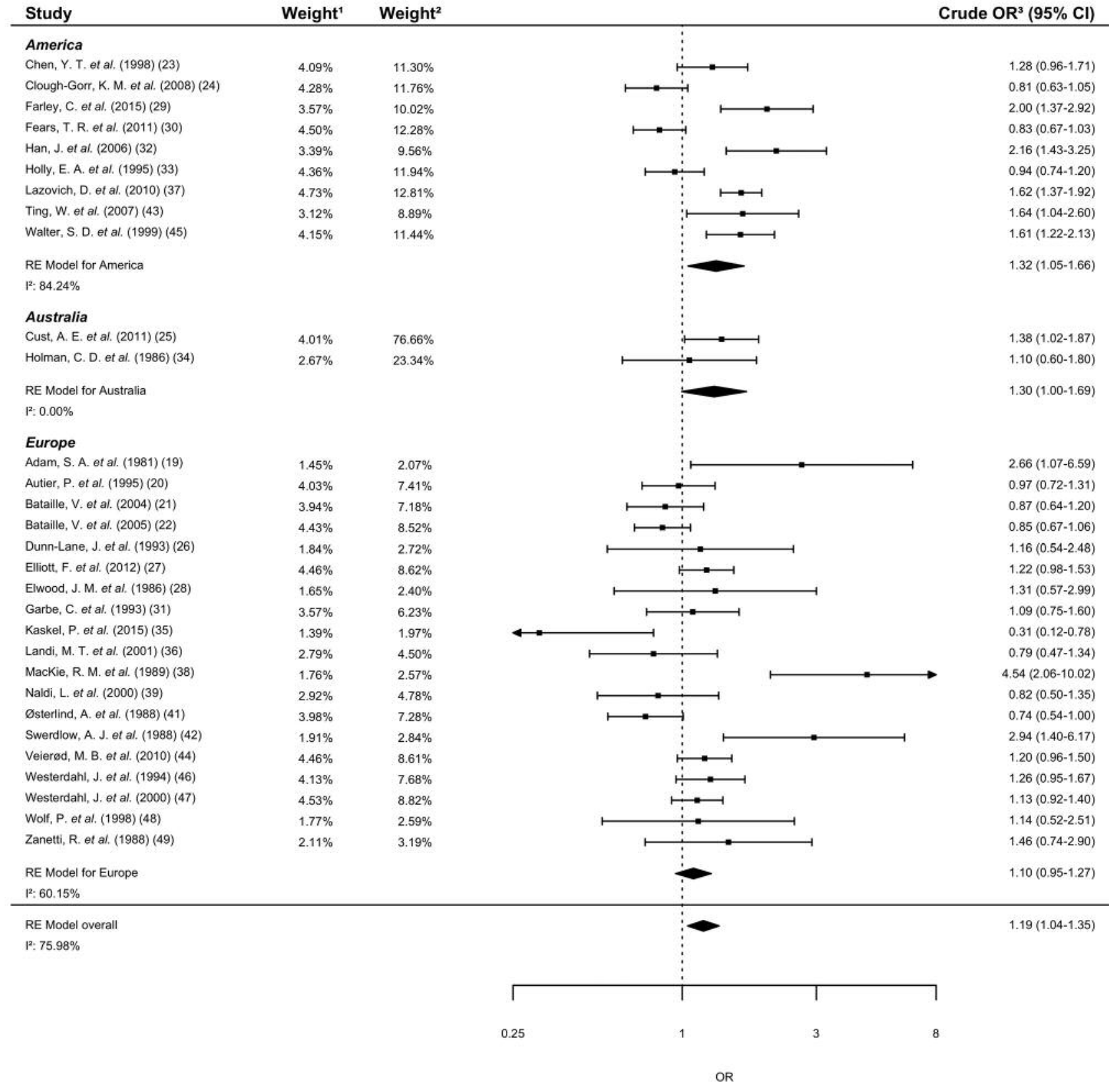

Figure 2. Forest plot for association of ever-exposure to UV radiation from a solarium with melanoma risk by geographic region. Rounding errors may occur in the forest plot. Weights refer to the ${ }^{1}$ overall summary risk estimate, ${ }^{2}$ summary risk estimates of respective subgroups; ${ }^{3}$ detailed information can be derived from Tables II, III. OR: Odds ratio; CI: confidence interval.

forced the authors to publish a correction (16). As Colantonio et al. point out, comparison of five previously published systematic reviews on this topic demonstrates an alarming tendency for copying data without referencing the original article, and without checking for errors (11). As an example, the influential review of the IARC Working group published in 2007 (10) has been criticized for numerous errors in content and typography [e.g. giving wrong numbers for the controls reported 1989 by MacKie et al. (38) and 1981 from Adam et al. (19)], which are also present in two subsequent reviews (11). Furthermore, the numbers of participants from several included studies $(31,43)$ published in the IARC review could not be derived by us and others (11) from the original articles. Our meta-analysis differs in several points 


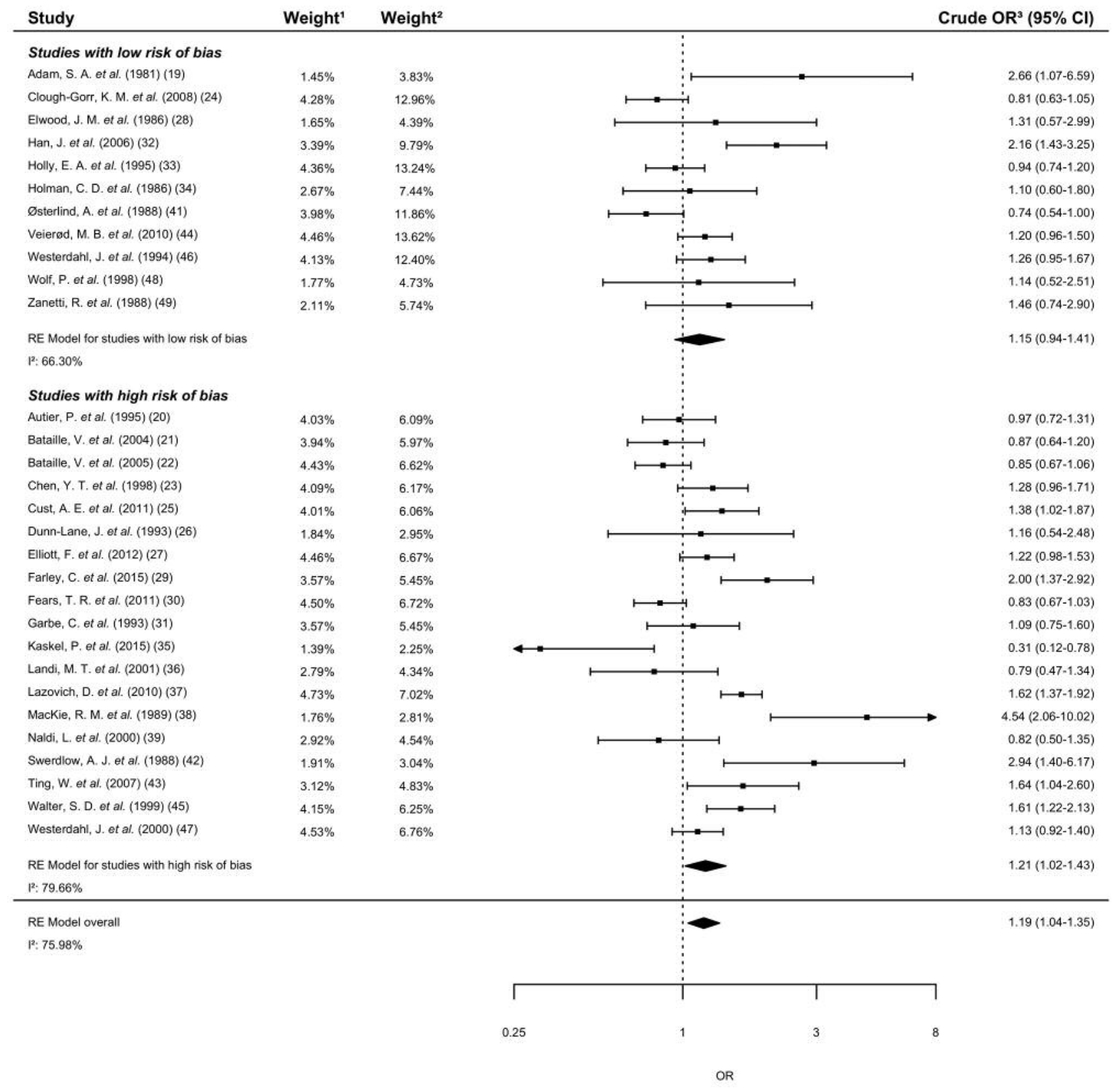

Figure 3. Forest plot for association of ever-exposure to UV radiation from a solarium with melanoma risk by risk of bias. Rounding errors may occur in the forest plot. Weights refer to the ${ }^{1}$ overall summary risk estimate, ${ }^{2}$ summary risk estimates of respective subgroups; ${ }^{3}$ detailed information can be derived from Tables II, III. OR: Odds ratio; CI: confidence interval.

from studies published previously. Firstly, we were able to identify and include some recently published studies that were not included in previous meta-analyses. Secondly, we chose a slightly different approach as compared with most meta-analyses published previously: because included casecontrol and cohort studies were heterogeneous regarding the adjustment of reported risk estimates (reporting crude/adjusted ORs or HRs; adjusted ORs or HRs were adjusted for greatly varying factors), we decided to use crude risk estimates in a random-effects model for our analysis to improve comparability. Moreover, we investigated the quality of individual studies using a modified Newcastle-Ottawa quality assessment scale and a generally accepted grading system for recommendations in evidence-based medicine. 
Table III. Summary risk estimates from random-effects meta-analyses.

\begin{tabular}{|c|c|c|c|c|c|c|c|c|c|}
\hline & $\begin{array}{l}\text { No. of } \\
\text { studies }\end{array}$ & $\begin{array}{c}\text { No. of } \\
\text { participants }\end{array}$ & $\begin{array}{l}\text { No. of } \\
\text { cases }\end{array}$ & $\begin{array}{c}\text { Crude OR } \\
(95 \% \mathrm{CI})\end{array}$ & $p$-Value & $\mathrm{I}^{2}$ & $\begin{array}{l}\text { Adjusted OR } \\
\quad(95 \% \mathrm{CI})\end{array}$ & $p$-Value & $\mathrm{I}^{2}$ \\
\hline & \multicolumn{9}{|c|}{ Ever exposure $v s$. non-exposure } \\
\hline Overall & 30 & 104,942 & 11,706 & $1.19(1.04-1.35)$ & 0.009 & $75.98 \%$ & $1.21(1.08-1.36)$ & 0.001 & $62.47 \%$ \\
\hline \multicolumn{10}{|l|}{ Study design } \\
\hline Case-control studies & 29 & 25,900 & 11,391 & $1.19(1.04-1.36)$ & 0.012 & $76.84 \%$ & $1.21(1.07-1.36)$ & 0.002 & $63.25 \%$ \\
\hline \multicolumn{10}{|l|}{ Geographic region } \\
\hline America & 9 & 10,229 & 4041 & $1.32(1.05-1.66)$ & 0.018 & $84.24 \%$ & $1.35(1.10-1.67)$ & 0.004 & $76.71 \%$ \\
\hline Australia & 2 & 1083 & 604 & $1.30(1.00-1.69)$ & 0.054 & $0.00 \%$ & $1.31(0.98-1.74)$ & 0.065 & $0.00 \%$ \\
\hline Europe & 19 & 93,630 & 7061 & $1.10(0.95-1.27)$ & 0.218 & $60.15 \%$ & $1.11(0.98-1.25)$ & 0.105 & $34.60 \%$ \\
\hline \multicolumn{10}{|l|}{ Year of recruitment } \\
\hline$\leq 1990$ & 13 & 8621 & 3896 & $1.33(1.07-1.66)$ & 0.010 & $69.35 \%$ & $1.21(1.01-1.45)$ & 0.040 & $49.20 \%$ \\
\hline$\geq 1991$ & 16 & 94,803 & 7731 & $1.09(0.93-1.29)$ & 0.295 & $79.51 \%$ & $1.19(1.02-1.38)$ & 0.027 & $69.60 \%$ \\
\hline 1991-1999 & 11 & 88,435 & 4243 & $0.98(0.82-1.17)$ & 0.816 & $66.67 \%$ & $1.11(0.94-1.31)$ & 0.233 & $51.41 \%$ \\
\hline$\geq 2000$ & 5 & 6368 & 3488 & $1.34(1.05-1.71)$ & 0.021 & $79.95 \%$ & $1.34(1.03-1.74)$ & 0.028 & $78.83 \%$ \\
\hline \multicolumn{10}{|l|}{ Risk of bias } \\
\hline Low $($ MNOS >4) & 11 & 85,219 & 2385 & $1.15(0.94-1.41)$ & 0.179 & $66.30 \%$ & $1.19(0.98-1.43)$ & 0.076 & $51.76 \%$ \\
\hline \multirow[t]{2}{*}{ High (MNOS $\leq 4)$} & 19 & 19,723 & 9321 & $1.21(1.02-1.43)$ & 0.029 & $79.66 \%$ & $1.22(1.06-1.41)$ & 0.007 & $66.09 \%$ \\
\hline & \multicolumn{9}{|c|}{ High exposure $v s$. non-exposure } \\
\hline \multirow[t]{2}{*}{ Overall } & 7 & 7691 & 3944 & $1.43(1.17-1.74)$ & $<0.001$ & $60.87 \%$ & $1.39(1.08-1.80)$ & 0.011 & $67.45 \%$ \\
\hline & \multicolumn{9}{|c|}{ Low exposure $v s$. non-exposure } \\
\hline \multirow[t]{2}{*}{ Overall } & 7 & 6995 & 3451 & $1.13(0.93-1.38)$ & 0.220 & $58.51 \%$ & $1.13(0.92-1.39)$ & 0.240 & $56.49 \%$ \\
\hline & \multicolumn{9}{|c|}{ First exposure at young age $v s$. non-exposure } \\
\hline Overall & 4 & 4602 & 2537 & $1.59(1.38-1.83)$ & $<0.001$ & $0.00 \%$ & $1.52(1.23-1.89)$ & $<0.001$ & $38.06 \%$ \\
\hline
\end{tabular}

Rounding errors may occur in data table. Total numbers of participants and cases are based on crude risk estimations and may differ for adjusted risk estimations. Summary adjusted risk estimates are based on estimates adjusted for the maximum number of covariates (crude risk estimates were used for studies without adjustment). The study of Ting et al. (43) was excluded from subgroup analyses regarding the year of recruitment due to missing information. High and low exposure to UV radiation from a solarium were defined as $>10$ and $\leq 10$ sessions in lifetime, respectively. First exposure to UV radiation from a solarium at young age refers to exposure before age 25 years.

The overall evidence level and quality of studies identified (19-49) was very low due to the lack of interventional trials and because of severe limitations of many of the observational studies. In the meta-analysis of all cohort and case-control studies identified by our literature search, we found a weak association for ever-exposure to UV radiation from a solarium with melanoma risk. Based on 27 studies, the meta-analysis of Boniol et al. (8) reported in 2012 a summary relative risk of 1.20 (95\% CI=1.08-1.34) for the association of ever-exposure to UV radiation from sunbeds with melanoma risk (heterogeneity: $\mathrm{I}^{2}=56 \%$ ). The authors also estimated that $3,438(5.4 \%)$ of 63,942 new cases of cutaneous malignant melanoma diagnosed each year in the 15 countries that were members of the European Community and the three countries that were part of the European Free Trade Association were related to sunbed use (8). Wehner $e t$ al. estimated the population proportional attributable risk of 2.6\%-9.4\% for melanoma, corresponding to more than 10,000 melanoma cases (12) each year attributable to solarium use in the United States, Europe and Australia. Colantonio et al. reported in their meta-analysis of 31 studies with data available on 14,956 melanoma cases and 233,106 controls an overall OR of 1.16 (95\% CI=1.05-1.28) for the association of ever-use of a solarium with melanoma risk (11). While the overall OR of our study $(\mathrm{OR}=1.19 ; 95 \%$ $\mathrm{CI}=1.04-1.35, p=0.009)$ is comparable with the results of Boniol et al. (8) and Colantonio et al. (11), we disagree with their conclusions. In our view, Boniol et al. (8) and Colantonio et al. (11) did not adequately consider the many limitations of the individual studies and the resulting low levels of evidence and grades of recommendation that do not allow postulation of a causal relationship between solarium use and melanoma risk. Moreover, in our opinion, the attempts of Boniol et al. (8) and others (12) to attribute melanoma cases to solarium use are speculative and scientifically not sufficiently supported.

Additionally, our meta-analysis indicated a moderate association of first exposure at younger age and high exposure to UV radiation from a solarium with melanoma risk. However, these results should be interpreted with caution. It should be emphasized that all cohort and casecontrol studies included in this meta-analysis (19-49) are likely to have overestimated the association of solarium use with melanoma risk in the general population because of 


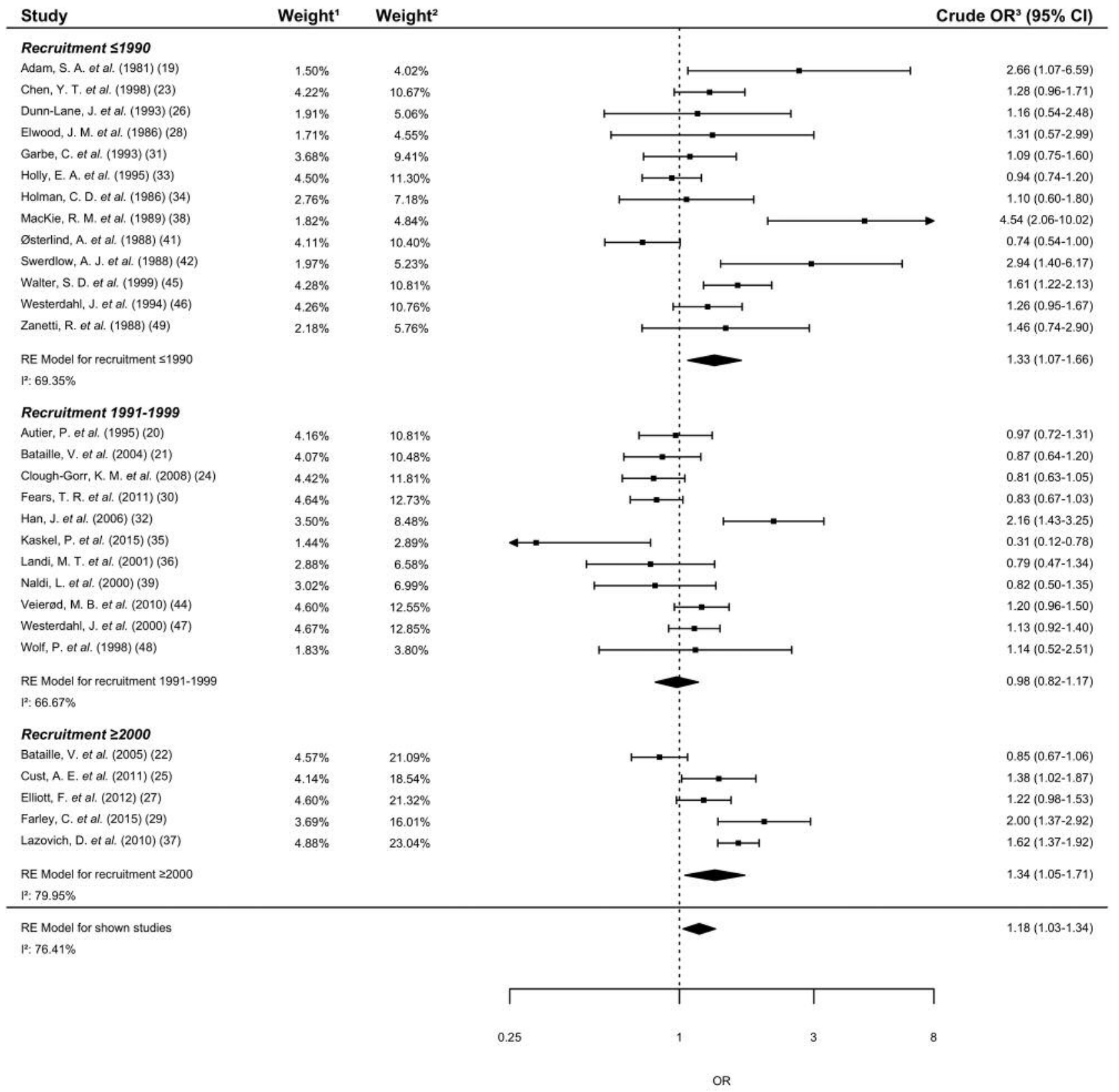

Figure 4. Forest plot for association of ever-exposure to UV radiation from a solarium with melanoma risk by recruitment period. Rounding errors may occur in the forest plot. Weights refer to the ${ }^{1}$ overall summary risk estimate, ${ }^{2}$ summary risk estimates of respective subgroups; ${ }^{3}$ detailed information can be derived from Tables II, III. OR: Odds ratio; CI: confidence interval.

many independent reasons, including (i) selection bias (exclusion of individuals with a likely relatively high UVexposure in the past [e.g. history of any kind of skin cancer or dermatological conditions] in controls, but not in cases), (ii) information bias (e.g. recall bias, the inclusion of nonsolarium exposure to artificial UV, e.g. phototherapy), (iii) difficulties in appropriately considering or adjusting for other confounding factors (e.g. solar UV or lifestyle, including smoking), and (iv) the restriction of the analysis to a subgroup of the general population, which may have an increased risk for melanoma (e.g. women).

Like others (37), our study could not confirm the emphasis of the IARC report (10) and of the report by Boniol et al. (8) on an increased melanoma risk with first use of indoor tanning 

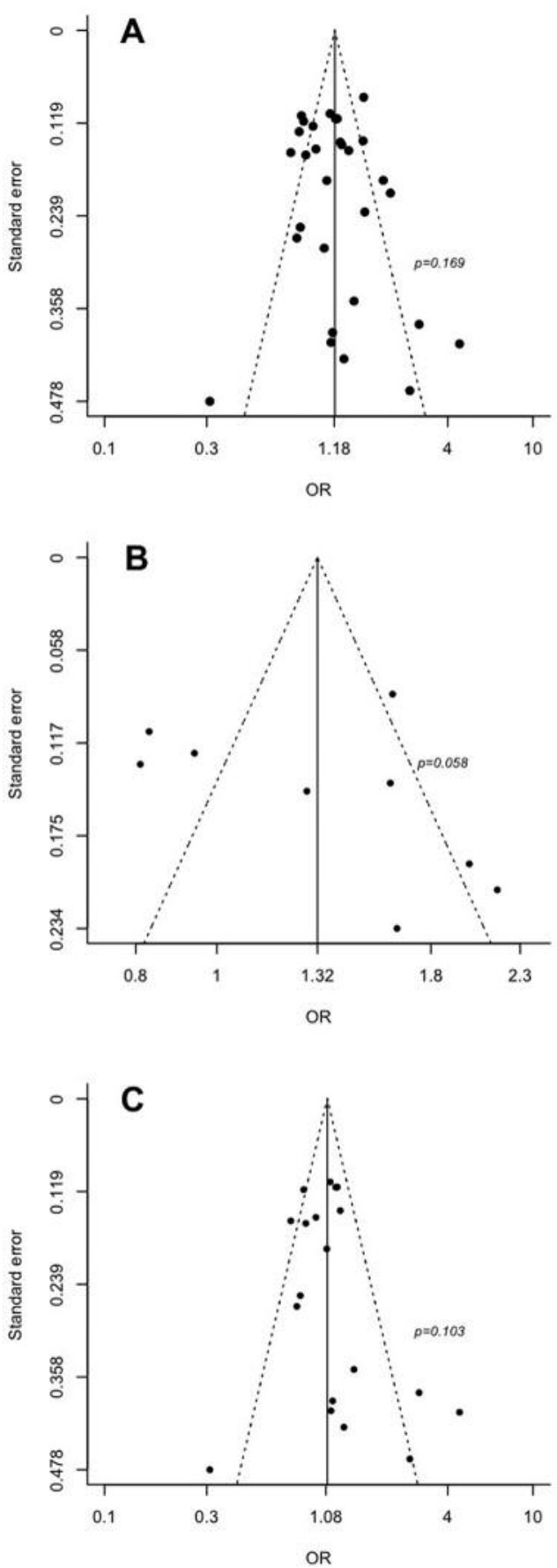

Figure 5. Funnel plots for association of ever-exposure to UV radiation from a solarium with melanoma risk. Notes: Funnel plot asymmetry was tested using Egger's test. A: All included studies $(n=31), B$ : studies from North America (n=9), C: studies from Europe $(n=20)$.

in younger age. It should be mentioned that both the IARC report (10) and the report by Boniol et al. (8) have to be criticized for defining first use in younger age as first use before the age of 36 years, but included studies that consider first use prior to ages 25 to 30 years $(23,42,55)$. Moreover, some studies $(46,47)$ restricted their investigation to melanoma cases diagnosed before the age of 36 years. However, this could have resulted in the exclusion of older cases and controls that may have been exposed at a younger age (37).

The obvious difficulties in considering or adjusting for important confounders are underlined in the Results section. Interestingly, subgroup analyses for studies performed in Europe, studies with low risk of bias, and studies with recruitment 1991-1999 did not show an association of melanoma risk with solarium use.

Concerning our finding of no significant statistical association between ever-exposure to UV radiation from a solarium and melanoma risk in studies performed in Europe (in contrast to studies performed in the United States, several factors are of particular relevance. Firstly, the role of solar UV exposure represents a major confounding factor that is difficult to control or to adjust for, and that may well at least in part explain latitude-dependent variations in melanoma risk. On the other hand, other region-specific factors, which include technical differences in solarium devices, must also be taken into account. Since 2008, solarium devices in Europe and Oceania (Australia and New Zealand) are restricted in intensity to an ultraviolet index of 12 and 36 (which was 60 before 2002), respectively. In contrast, the intensity of a solarium in the United States is unlimited but often a "maximum recommended exposure time" is given.

For many factors that may influence the association of solarium use and melanoma risk, including legal regulations, solarium technology and epidemiology of solarium use, which are subject to frequent change, it is of particular interest to evaluate trends over time. Another interesting observation of our sensitivity and subgroup analyses was the finding that recruitment period had a strong impact on the association of melanoma risk with solarium use. For recruitment before 1991, a higher OR was found as compared with recruitment from 1991-1999 or since 2000. It can be speculated that this observation is due to changes in operation and technical modifications of UV-emitting devices (approximately two decades ago, the solarium industry started to produce devices with higher pressure bulbs emitting larger doses of long-wave UV A). The results of our meta-analysis and previous published studies most likely overestimate the association of melanoma risk with current solarium use as many countries have recently imposed strict regulations on solarium use that, besides other effects, should reduce first use at younger age and high use of a solarium. However, the questions whether stricter regulations of recent years and technical progress have further improved the safety of solarium use are difficult to answer because in many cases, solarium use is not clearly restricted to distinct time periods of interest. 
We emphasize that interventional trials are lacking and that the results of the cohort and case-control studies included in this meta-analysis represent associations that do not prove causality. Moreover, both the resulting level of evidence and grade of recommendation of studies investigating the association of melanoma risk with solarium use are weak. According to the Oxford Centre for Evidence-based Medicine, for all outcomes analyzed in our meta-analysis, we found level 3a- of evidence (poor quality cohort and casecontrol studies) and grade D of recommendation. The poor quality of the cohort and case-control studies included in this meta-analysis is due to severe limitations that include difficulties in appropriately considering and controlling for known confounders (e.g. exposure with solar UV or artificial UV for medical purposes; lifestyle, including smoking).

In summary, our review has highlighted the poor quality of the evidence available at present on this topic. We conclude that (i) results of our and previously published meta-analyses most likely overestimated the association of melanoma risk with solarium use, (ii) both the level of evidence and grade of recommendation of studies published previously investigating the association of melanoma risk with solarium use are weak, and therefore (iii) present scientific knowledge does not support the hypothesis of an increased melanoma risk due to solarium use, and questions studies that try to attribute melanoma cases to indoor tanning, and does not support initiatives that aim to ban responsible/moderate solarium use for tanning purposes.

\section{Author's Disclosures}

BB, JS, IH, CS, SR, SW, SP, JOM, WM have nothing to disclose. Saarland University (TV, JR) received funding from Jörg Wolff Foundation and Immundiagnostik AG. JR received speakers honoraria from Wörwag and Dermapharm.

\section{References}

1 Reichrath J: The challenge resulting from positive and negative effects of sunlight: how much solar UV exposure is appropriate to balance between risks of vitamin D deficiency and skin cancer? Prog Biophys Mol Biol 92: 9-16, 2006.

2 Holick MF: Vitamin D deficiency. N Engl J Med 357: 266-281, 2007.

3 Grant WB, Garland CF and Holick MF: Comparisons of estimated economic burdens due to insufficient solar ultraviolet irradiance and vitamin D and excess solar UV irradiance for the United States. Photochem Photobiol 81: 1276-1286, 2005.

4 Holick MF: Sunlight "D"ilemma: risk of skin cancer or bone disease and muscle weakness. Lancet 357: 4-6, 2001.

5 Reichrath J and Rass K: Ultraviolet damage, DNA repair and vitamin $\mathrm{D}$ in nonmelanoma skin cancer and in malignant melanoma: an update. Adv Exp Med Biol 810: 208-233, 2014.

6 Mason RS and Reichrath J: Sunlight vitamin D and skin cancer. Anticancer Agents Med Chem 13: 83-97, 2013.
7 Autier P, Koechlin A and Boniol M: The forthcoming inexorable decline of cutaneous mortality in light-skinned populations. Eur J Cancer 51: 869-878, 2015.

8 Boniol M, Autier P, Boyle P and Gandini S: Cutaneous melanoma attributable to sunbed use: systematic review and meta-analysis. BMJ 345: e4757, 2012.

9 El Ghissassi F, Baan R, Straif K, Grosse Y, Secretan B, Bouvard V, Benbrahim-Tallaa L, Guha N, Freeman C, Galichet L, Cogliano $\mathrm{V}$ and WHO International Agency for Research on Cancer Monograph Working Group: A review of human carcinogens - part D: radiation. Lancet Oncol 10(8): 751-752, 2009.

10 IARC Working Group on Artificial UV light and skin cancer: The association of use of sunbeds with cutaneous malignant melanoma and other skin cancers: a systematic review. Int $\mathbf{J}$ Cancer 120: 1116-1122, 2007.

11 Colantonio S, Bracken MB and Beecker J: The association of indoor tanning and melanoma in adults: systematic review and meta-analysis. J Am Acad Dermatol 70: 847-857, 2014.

12 Wehner MR, Chren MM, Nameth D, Choudhry A, Gaskins M, Nead KT, Boscardin WJ and Linos E: International prevalence of indoor tanning: a systematic review and meta-analysis. JAMA Dermatol 150: 390-400, 2014.

13 Gallagher RP, Spinelli JJ and Lee TK: Tanning beds, sunlamps, and risk of cutaneous malignant melanoma. Cancer Epidemiol Biomarkers Prev 14(3): 562-566, 2005.

14 Hirst N, Gordon L, Gies P and Green AC: Estimation of avoidable skin cancers and cost-savings to government associated with regulation of the solarium industry in Australia. Health Policy 89(3): 303-311, 2009.

15 Swerdlow AJ and Weinstock MA: Do tanning lamps cause melanoma? An epidemiologic assessment. J Am Acad Dermatol 38(1): 89-98, 1998.

16 Boniol M, Autier P, Boyle $\mathrm{P}$ and Gandini S: Cutaneous melanoma attributable to sunbed use: systematic review and meta-analysis. BMJ 345: e8503 (correction), 2012.

17 Grant WB: Critique of the IARCs meta-analyses of the association of sunbed use with risk of cutaneous malignant melanoma. Dermato-Endocrinology 1: 294-299, 2009.

18 Moan JE, Baturaite Z, Grigalavicius M and Juzeniene A: Sunbed use and cutaneous melanoma in Norway. Scand J Public Health 41(8): 812-817, 2013.

19 Adam SA, Sheaves JK, Wright NH, Mosser G, Harris RW and Vessey MP: A case-control study of the possible association between oral contraceptives and malignant melanoma. $\mathrm{Br} \mathrm{J}$ Cancer 44(1): 45-50, 1981.

20 Autier P, Dore JF, Schifflers E, Cesarini JP, Bollaerts A, Koelmel KF, Gefeller O, Liabeuf A, Lejeune F and Lienard D: Melanoma and use of sunscreens: an Eortc case-control study in Germany, Belgium and France. The EORTC Melanoma Cooperative Group. Int J Cancer 61(6): 749-755, 1995.

21 Bataille V, Winnett A, Sasieni P, Newton Bishop JA and Cuzick $\mathrm{J}$ : Exposure to the sun and sunbeds and the risk of cutaneous melanoma in the UK: a case-control study. Eur J Cancer 40(3): 429-435, 2004

22 Bataille V, Boniol M, De Vries E, Severi G, Brandberg Y, Sasieni P, Cuzick J, Eggermont A, Ringborg U, Grivegnée AR, Coebergh JW, Chignol MC, Doré JF and Autier P: A multicentre epidemiological study on sunbed use and cutaneous melanoma in Europe. Eur J Cancer 41(14): 2141-2149, 2005. 
23 Chen YT, Dubrow R, Zheng T, Barnhill RL, Fine J and Berwick M: Sunlamp use and the risk of cutaneous malignant melanoma: a population-based case-control study in Connecticut, USA. Int J Epidemiol 27(5): 758-765, 1998.

24 Clough-Gorr KM, Titus-Ernstoff L, Perry AE, Spencer SK and Ernstoff MS: Exposure to sunlamps, tanning beds, and melanoma risk. Cancer Causes Control 19(7): 659-669, 2008.

25 Cust AE, Armstrong BK, Goumas C, Jenkins MA, Schmid H, Hopper JL, Kefford RF, Giles GG, Aitken JF and Mann GJ: Sunbed use during adolescence and early adulthood is associated with increased risk of early-onset melanoma. Int $\mathrm{J}$ Cancer 128(10): 2425-2435, 2011.

26 Dunn-Lane J, Herity B, Moriarty MJ and Conroy R: A case control study of malignant melanoma. Ir Med J 86(2): 57-59, 1993.

27 Elliott F, Suppa M, Chan M, Leake S, Karpavicius B, Haynes S, Barrett JH, Bishop DT and Newton-Bishop JA: Relationship between sunbed use and melanoma risk in a large case-control study in the United Kingdom. Int J Cancer 130(12): 3011-3013, 2012.

28 Elwood JM, Williamson C and Stapleton PJ: Malignant melanoma in relation to moles, pigmentation, and exposure to fluorescent and other lighting sources. Br J Cancer 53: 65-74, 1986.

29 Farley C, Alimi Y, Espinosa LR, Perez S, Knechtle W, Hestley A, Carlson GW, Russell MC, Delman KA and Rizzo M: Tanning beds: A call to action for further educational and legislative efforts. J Surg Oncol 112(2): 183-187, 2015.

30 Fears TR, Sagebiel RW, Halpern A, Elder DE, Holly EA, Guerry D 4th and Tucker MA: Sunbeds and sunlamps: who used them and their risk for melanoma. Pigment Cell Melanoma Res 24(3): 574-581, 2011.

31 Garbe C, Weiss J, Kruger S, Garbe E, Büttner P, Bertz J, Hoffmeister H, Guggenmoos-Holzmann I, Jung EG and Orfanos CE: The German melanoma registry and environmental risk factors implied. Recent Results Cancer Res 128: 69-89, 1993.

32 Han J, Colditz GA and Hunter DJ: Risk factors for skin cancers: a nested case-control study within the Nurses' Health Study. Int J Epidemiol 35: 1514-1521, 2006.

33 Holly EA, Aston DA, Cress RD, Ahn DK and Kristiansen JJ: Cutaneous melanoma in women. I. Exposure to sunlight, ability to tan, and other risk factors related to ultraviolet light. Am J Epidemiol 141(10): 923-933, 1995.

34 Holman CD, Armstrong BK, Heenan PJ, Blackwell JB, Cumming FJ, English DR, Holland S, Kelsall GR, Matz LR and Rouse IL: The causes of malignant melanoma: results from the West Australian Lions Melanoma Research Project. Recent Results Cancer Res 102: 18-37, 1986.

35 Kaskel P, Lange U, Sander S, Huber MA, Utikal J, Leiter U, Krähn G, Meurer M and Kron M: Ultraviolet exposure and risk of melanoma and basal cell carcinoma in Ulm and Dresden, Germany. J Eur Acad Dermatol Venereol 29(1): 134-142, 2015.

36 Landi MT, Baccarelli A, Calista D, Pesatori A, Fears T, Tucker MA and Landi G: Combined risk factors for melanoma in a Mediterranean population. Br J Cancer 85(9): 1304-1310, 2001.

37 Lazovich D, Vogel RI, Berwick M, Weinstock MA, Anderson $\mathrm{KE}$ and Warshaw EM: Indoor tanning and risk of melanoma: a case-control study in a highly exposed population. Cancer Epidemiol Biomarkers Prev 19(6): 1557-1568, 2010.
38 MacKie RM, Freudenberger T and Aitchison TC: Personal risk-factor chart for cutaneous melanoma. Lancet 2: 487-490, 1989.

39 Naldi L, Gallus S, Imberti GL, Cainelli T, Negri E and La Vecchia C: Sunlamps and sunbeds and the risk of cutaneous melanoma. Italian Group for Epidemiological Research in Dermatology. Eur J Cancer Prev 9(2): 133-134, 2000.

40 Nielsen K, Masback A, Olsson H and Ingvar C: A prospective, population-based study of 40,000 women regarding host factors, UV exposure and sunbed use in relation to risk and anatomic site of cutaneous melanoma. Int J Cancer 131(3): 706-715, 2012.

41 Osterlind A, Tucker MA, Stone BJ and Jensen OM: The Danish case-control study of cutaneous malignant melanoma. II. Importance of UV-light exposure. Int J Cancer 42(3): 319-324, 1988.

42 Swerdlow AJ, English JS, MacKie RM, O'Doherty CJ, Hunter JA, Clark J and Hole DJ: Fluorescent lights, ultraviolet lamps, and risk of cutaneous melanoma. BMJ 297(6649): 647-650; 1988. Erratum in: BMJ 297(6657): 1172, 1988.

43 Ting W, Schultz K, Cac NN, Peterson M and Walling HW: Tanning bed exposure increases the risk of malignant melanoma. Int J Dermatol 46(12): 1253-1257, 2007.

44 Veierod MB, Adami HO, Lund E, Armstrong BK and Weiderpass E: Sun and solarium exposure and melanoma risk: effects of age, pigmentary characteristics, and nevi. Cancer Epidemiol Biomarkers Prev 19(1): 111-120, 2010.

45 Walter SD, King WD and Marrett LD: Association of cutaneous malignant melanoma with intermittent exposure to ultraviolet radiation: results of a case-control study in Ontario, Canada. Int J Epidemiol 28: 418-427, 1999.

46 Westerdahl J, Olsson H, Masback A, Ingvar C, Jonsson N, Brandt L, Jönsson PE and Möller T: Use of sunbeds or sunlamps and malignant melanoma in southern Sweden. Am J Epidemiol 140(8): 691-699, 1994.

47 Westerdahl J, Ingvar C, Masback A, Jonsson $\mathrm{N}$ and Olsson $\mathrm{H}$ : Risk of cutaneous malignant melanoma in relation to use of sunbeds: further evidence for UV-A carcinogenicity. Br J Cancer 82(9): 1593-1599, 2000.

48 Wolf P, Quehenberger F, Mullegger R, Stranz B and Kerl H: Phenotypic markers, sunlight-related factors and sunscreen use in patients with cutaneous melanoma: an Austrian case-control study. Melanoma Res 8(4): 370-378, 1998.

49 Zanetti R, Rosso S, Faggiano F, Roffino R, Colonna S and Martina G: A case-control study of melanoma of the skin in the province of Torino, Italy. Rev Epidemiol Sante Publique 36(45): 309-317, 1988.

50 Zivkovic MV, Dediol I, Ljubicic I and Situm M: Sun behaviour patterns and perception of illness among melanoma patients. J Eur Acad Dermatol Venereol 26(6): 724-729, 2012.

51 Beitner H, Norell SE, Ringborg, Wennersten G and Mattson B: Malignant melanoma: aetiological importance of individual pigmentation and sun exposure. Br J Dermatol 122(1): 43-51, 1990.

52 Gallagher RP, Elwood JM and Hill GB: Risk factor for cutaneous malignant melanoma: The Western Canada Melanoma Study. Recent Results Cancer Res 102: 38-55, 1986.

53 Holly EA, Kelly JW, Shpall SN and Chiu SH: Number of melanocytic nevi as a major risk factor for malignant melanoma. J Am Acad Dermatol 17(3): 459-468, 1987. 
54 Klepp $\mathrm{O}$ and Magnus K: Some environmental and bodily characteristics of melanoma patients. A case-control study. Int J Cancer 23(4): 482-486, 1979.

55 Schmitt J, Seidler A, Heinisch G and Sebastian G: Effectiveness of skin cancer screening for the age group 14 through 34 years. J Dtsch Dermatol Ges 9: 608-617, 2011.

56 Ferrucci LM, Vogel RI, Cartmel B, Lazovich D and Mayne ST: Indoor tanning in businesses and homes and risk of melanoma and nonmelanoma skin cancer in 2 US case-control studies. J Am Acad Dermatol 71(5): 882-887, 2014.

57 Veierod MB, Weiderpass E, Thörn M, Hansson J, Lund E, Armstrong $B$ and Adami HO: A prospective study of pigmentation, sun exposure, and risk of cutaneous malignant melanoma in women. J Natl Cancer Inst 95(20): 1530-1538, 2003.

58 Walter SD, Marrett LD, From L, Hertzman C, Shannon HS and Roy P: The association of cutaneous malignant melanoma with the use of sunbeds and sunlamps. Am J Epidemiol 131(2): 232243, 1990.

59 Zhang M, Qureshi AA, Geller, AC, Frazier L, Hunter DJ and Han J: Use of tanning beds and incidence of skin cancer. J Clin Oncol 30(14): 1588-1593, 2012.
60 Moher D, Liberati A, Tetzlaff J, Altman DG and PRISMA Group: Preferred reporting items for systematic reviews and meta-analyses: the PRISMA statement. BMJ 339: b2535, 2009.

61 Stroup DF, Berlin JA, Morton SC, Olkin I, Williamson GD, Rennie D, Moher D, Becker BJ, Sipe TA and Thacker SB: Metaanalysis of observational studies in epidemiology (MOOSE) Group. Meta-analysis of observational studies in epidemiology: a proposal for reporting. JAMA 283: 2008-2012, 2000.

$62 \mathrm{http} / / / \mathrm{cccrg}$.cochrane.org/author-resources (last access: March $22,2016)$

63 http://www.ohri.ca/programs/clinical_epidemiology/nosgen.doc (last access: March 22, 2016).

64 htpp://www.cebm.net/oxford-centre-evidence-based-medicinelevels-evidence-march-2009/ (last access: December 15, 2017)

Received October 31, 2017

Revised December 13, 2017

Accepted December 29, 2017 